\title{
ARQUEOSISMOLOGÍA: UNA NUEVA HERRAMIENTA PARA LA SISMO- LOGÍA Y LA PROTECCIÓN DEL PATRIMONIO
}

\author{
M.A. Rodríguez-Pascua1', P.G. Silva², J.L. Giner-Robles³, R. Pérez-López', \\ M.A. Perucha ${ }^{1}$, y F. Martín-González ${ }^{4}$
}

\section{Resumen:}

La Arqueosismología es una técnica multidisciplinar enfocada al estudio de terremotos en el pasado histórico mediante yacimientos arqueológicos y patrimonio cultural. Los datos procedentes de la arqueología son fundamentales a la hora de poder realizar interpretaciones arqueosismológicas, esto hace que la colaboración interdisciplinar Arqueología-Geología sea fundamental a la hora de elaborar conclusiones fiables. La inclusión del análisis estructural geológico en el estudio de las deformaciones sísmicas del patrimonio cultural, permiten conocer cuál ha sido el origen de las mismas y cómo se comportará este patrimonio en el futuro. También puede aportar nuevos datos a los catálogos sísmicos, con terremotos no registrados documentalmente. La arqueosismología se muestra como una herramienta adecuada para aplicar en la prevención contra terremotos que pueden afectar a nuestro patrimonio cultural.

\section{Palabras clave:}

Terremoto, Arqueosismología, Efectos Arqueológicos de Terremotos (EAEs), Análisis Estructural Geológico.

\section{Abstract:}

The Archaeoseismology is a multidisciplinary technique focused in the study of the historical earthquakes by archaeological sites and cultural heritage. The data from the Archaeology are fundamental to make archaeoseismic interpretations, for this reason the interdisciplinary collaboration Archaeology-Geology is essential in order to make reliable conclusions. The inclusion of structural geological analysis in the study of seismic deformations of cultural heritage offers the possibility to know the origin of such deformation and how the heritage will behave in the future. The Archaeoseismology can also add new data to the seismic catalogues with unregistered earthquakes. The Archaeoseismology is an appropriate tool to apply for seismic prevention affecting of our cultural heritage.

1 Instituto Geológico y Minero de España. Madrid. España.

ma.rodriguez@igme.es, r.perez@igme.es, ma.perucha@igme.es

2 Dpto. Geología, Escuela Politécnica Superior de Ávila, Universidad Salamanca. Ávila. España. pgsilva@usal.es

3 Facultad de Ciencias, Universidad Autónoma de Madrid. Madrid. SPAIN. jorge.giner@uam.es 4 Área de Geología, ESCET, Universidad Rey Juan Carlos. Móstoles (Madrid). España.

fidel.martin@urjc.es 


\section{Key words:}

Earthquake, Archaeoseismology, Earthquake Archaeological Effects (EAEs), Geological Structural Analysis.

\section{Introducción}

Podríamos definir la arqueosismología como la disciplina encargada del estudio de los terremotos a través del estudio de sus efectos extraídos de yacimientos arqueológicos y/o patrimonio cultural. A pesar de que la arqueosismología empezó a crearse a finales del s. XIX, en los últimos años, ya ha pasado de ser un modismo a una disciplina conocida y aplicada en el ámbito del estudio de los terremotos. Lyell en su "Principios de Geología" de 1872, ya cita varios casos de excavaciones arqueológicas utilizadas para determinar la presencia de terremotos en el pasado. Los diferentes esfuerzos realizados por múltiples autores desde los años 80’ (Rapp, 1982; Zang et al., 1986; Stiros, 1988a y b; Nikonov, 1988; Guidoboni, 1989) han hecho que esta técnica multidisciplinar sea conocida en el ámbito de la sismología y comience a ser tenida en cuenta, aunque otros autores ya habían introducido con anterioridad los terremotos en las interpretaciones arqueológicas (Lanciani, 1918).

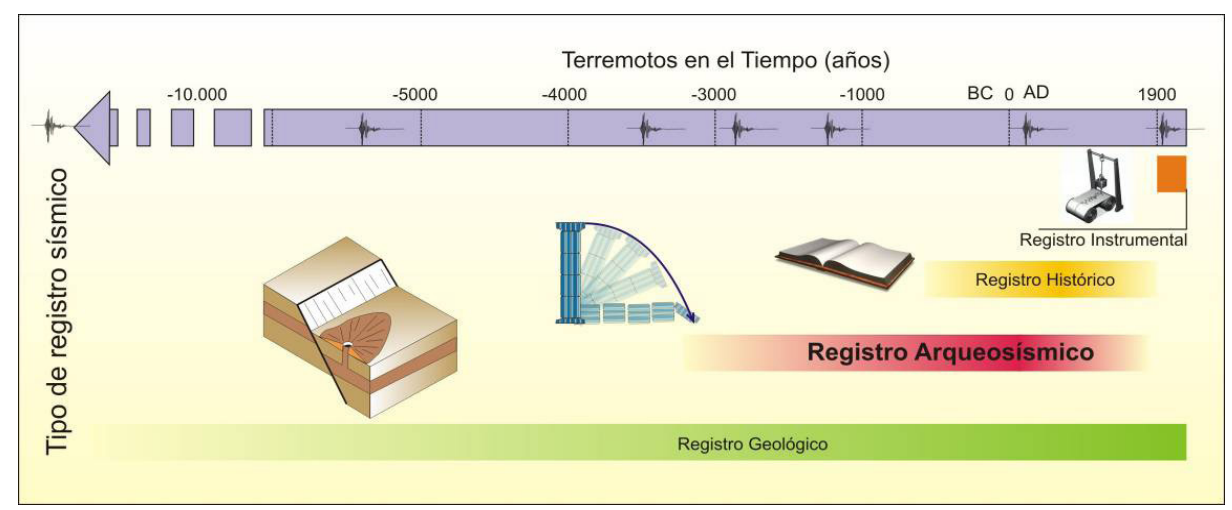

Fig. 1. Intervalo temporal ocupado por el registro arqueosismológico.

Cuando en los años 90’ se realiza una recopilación del estado del conocimiento Ilevada a cabo por Stiros y Jones (1996), coinciden tanto en la necesidad de la colaboración multidisciplinar entre arqueología, sismología, geología, ingeniería, arquitectura e historia, así como, en preguntarse ¿Qué criterios son necesarios para identificar efectos sísmicos en un yacimiento arqueológico? La primera premisa está ampliamente aceptada y el trabajo multidisciplinar es absolutamente necesario para poder avanzar en el estado del conocimiento en 
arqueosismología. El rango temporal que abarca la arqueosismología depende de la zona en la que nos encontremos (Fig. 1). Sobre todo es útil desde la aparición de las primeras construcciones humanas susceptibles de ser afectadas por terremotos, aunque también se han registrado en ocasiones colapsos en cuevas atrapando Homo sapiens o neandertales, por lo que el registro podría irse hasta el Pleistoceno. En el caso de España el registro puede ir hasta la Edad del Bronce, pero para ser utilizado con ciertas garantías no podríamos ir más allá de la romanización (s. III BC).

En cuanto a los criterios a tener en cuenta para discriminar el origen sísmico de las deformaciones encontradas en un yacimiento arqueológico, aún son objeto de debate científico. En este sentido han surgido trabajos como el de Sintubin y Stewart (2008) en el que proponen una metodología con estructura de árbol lógico para cuantificar un factor del potencial arqueosísmico de un yacimiento arqueológico. Pero para poder discriminar el origen sísmico de estructuras de deformación en yacimientos arqueológicos es necesario aplicar una metodología científica; para lo cual, lo primero que se necesita es una clasificación de estructuras en función de su origen. Rodríguez-Pascua et al. (2011) proponen una clasificación de Efectos Arqueológicos de Terremotos (Earthquake Archaeological Effects, EAEs) que cubre este paso inicial, además de incluir en el 2013 una escala de intensidades basada en la combinación de las escalas macrosísmicas EMS98 y la de efectos geológicos de terremotos ESI07. Una vez clasificados estos efectos se lleva a cabo un análisis estructural geológico para poder determinar si son estructuras que siguen patrones de deformación orientados por el movimiento del terreno o no. En este sentido, Giner Robles et al. (2011) editan una guía de metodológica de trabajo en este ámbito.

\section{Metodología}

La arqueosismología es un método científico multidisciplinar que tiene un doble objetivo: por un lado, el determinar terremotos no registrados históricamente, y por otro, el estudio del comportamiento sísmico del patrimonio cultural. Con todos estos datos, se abren nuevas posibilidades para el estudio de fallas activas mediante paleosismología, se obtienen nuevos registros para completar los catálogos sísmicos, para su aplicación a la peligrosidad y riesgo sísmico, y para la creación de protocolos de actuación para protección del patrimonio en caso de sismo, así como la toma de medidas preventivas. En el caso de la paleosismología el flujo metodológico es reversible, puesto que investigaciones paleosismológicas en fallas activas también nos pueden poner sobre la pista de yacimientos arqueológicos que pudieron ser afectados por estos paleoterremotos (Fig. 2).

Para poder identificar terremotos en el pasado histórico se puede recurrir a tres tipos de registro: documental, arqueológico y edificaciones/construcciones de interés patrimonial. 
Con toda esta información, y el trabajo de campo, podremos catalogar los diferentes EAEs. Los EAEs se muestran como buenos indicadores para la identificación de terremotos no registrados históricamente, permitiendo la cuantificación de la deformación y su aplicación a la protección del patrimonio cultural. Estas metodologías han sido calibradas con éxito en el reciente terremoto de Lorca (11/05/2011) (Giner Robles et al., 2012; Rodríguez-Pascua et al., 2012) y han sido utilizadas tanto en la estabilización de edificios históricos como en los planes de restauración (De la Hoz, 2012a y b).

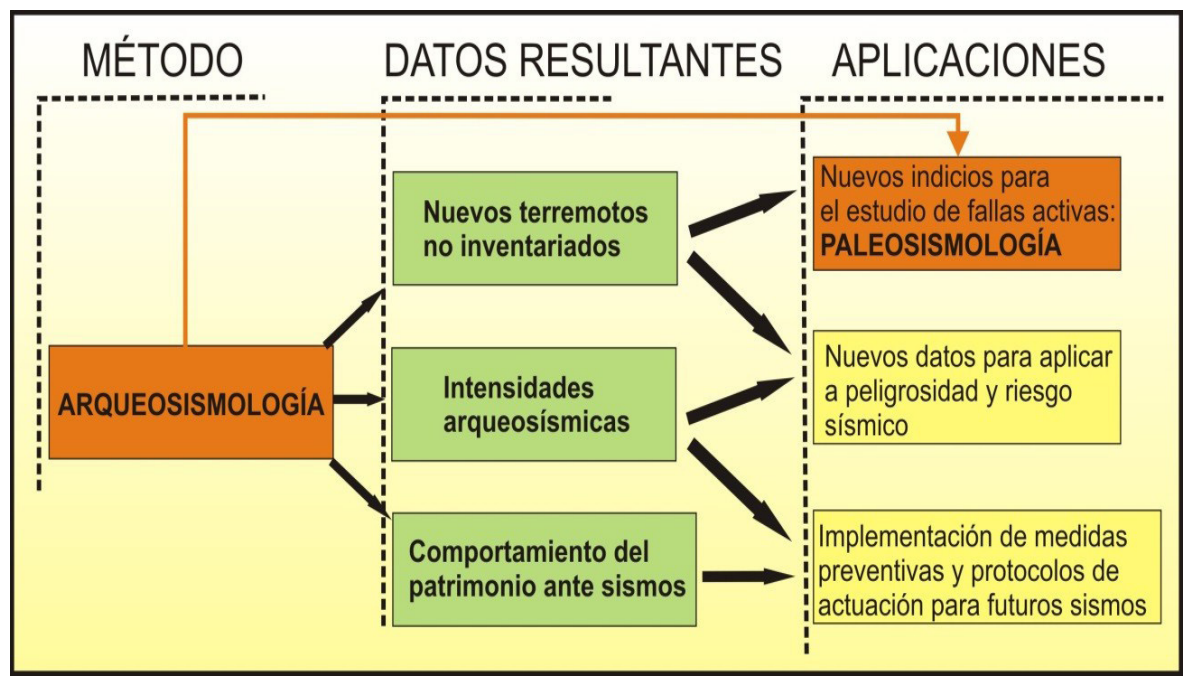

Figura 2. Aplicaciones y tipos de datos que generan los estudios arqueosismológicos.

Con toda la información previa recopilada se pasaría al estudio en detalle de las deformaciones que representan los EAEs, mediante análisis estructural geológico y/o modelización numérica mediante computación. El objetivo principal es calcular las orientaciones de las deformaciones de las construcciones y su relación, tanto con la falla que generó el terremoto, como con la llegada de las ondas sísmicas al yacimiento. Todos estos datos arqueosísmicos pueden ser corroborados y validados por técnicas paleosismológicas, las cuales van a permitir calcular los parámetros sismogenéticos de la falla que produjo el sismo, entre ellos la secuencia sísmica de los últimos terremotos que afectaron al yacimiento arqueológico.

El caso del terremoto de Lorca de 2011 también puso de manifiesto la necesidad de crear protocolos de intervención rápida, tanto desde un punto de vista de la protección del patrimonio, como de la toma de datos de EAEs, que permitan respaldar las intervenciones en la protección patrimonial (conclusiones de las Jornadas de expertos sobre Patrimonio en Riesgo que organizó el IPCE en Lorca; IPCE, 2012). Mientras escribimos estas líneas ha tenido lugar el terremoto de Nepal $(25 / 04 / 2015 ; M=7,8)$, donde las víctimas ya se cuentan por miles, habiendo 
quedado destruido gran parte del patrimonio cultural de este país, especialmente en su capital Katmandú. Cada nuevo terremoto nos hace reflexionar sobre la necesidad de avanzar en el conocimiento arqueosismológico para poder paliar en la medida de lo posible estas catástrofes. Todo esto indica el potencial de una disciplina que está emergiendo en la actualidad y que está respaldada por instituciones como INQUA (International Association for Quaternary Research) o la UNESCO mediante sus proyectos IGCP.

\section{Efectos arqueológicos de terremotos (earthquake archaeological effects, EAEs)}

En toda investigación arqueosismológica es necesario realizar una clasificación de EAEs previa a su estudio pormenorizado. Para este fin, Rodríguez-Pascua et al. (2011) proponen una clasificación de EAEs, basada en los efectos que se producen durante el terremoto y los que tienen lugar después del mismo; es decir, efectos cosísmicos y postsísmicos (Fig. 3). Los efectos cosísmicos se pueden dividir a su vez en efectos geológicos y efectos en la fábrica de los edificios. Dentro de los Efectos Arqueológicos de Terremotos (EAEs), los efectos postsísmicos o efectos indirectos, pueden dar pistas para iniciar una investigación arqueosísmica en un determinado yacimiento, pero no van a permitir determinar de forma directa la existencia de un terremoto en el pasado, como abandonos injustificados, reparaciones con elementos reciclados o construcciones antisísmicas (Fig. 4). Las descripciones históricas de terremotos también aportan datos de edificaciones importantes que sufrieron daños, como puedan ser catedrales o palacios. Esta información permite focalizar la búsqueda de deformaciones arqueosísmicas en el campo. Mucha de esta información ya está recopilada y seleccionada por parte de catálogos como el de Galbis (1932 y 1940) o el de Martínez Solares y Mezcua (2002). Sin embargo, existe más información referente a la especificación de daños en grandes construcciones, por ejemplo catedrales, cuyo contenido no ha sido objeto por parte de estos catálogos, centrados fundamentalmente en la identificación de eventos sísmicos, sin entrar en estos detalles. En algunos archivos catedralicios se puede encontrar información exhaustiva de estos efectos, como por ejemplo los daños producidos por el Terremoto de Lisboa (1755) en la Catedral de Coria (Martínez-Vázquez, 1999) o los efectos producidos en la Colegiata de San Patricio en Lorca por el terremoto de 1674 (Muñoz Clares et al., 2012). De este modo se puede tener un dato orientado de la deformación; dato que se puede contrastar observando los daños que han podido permanecer hasta nuestros días o las reparaciones de los mismos. En algunos casos también es posible encontrar información periodística con grabados de época en los que se puede extraer este tipo de deformaciones. Mención aparte merecen los terremotos acaecidos después de la invención de la fotografía, donde podemos observar de forma directa estos EAEs. 


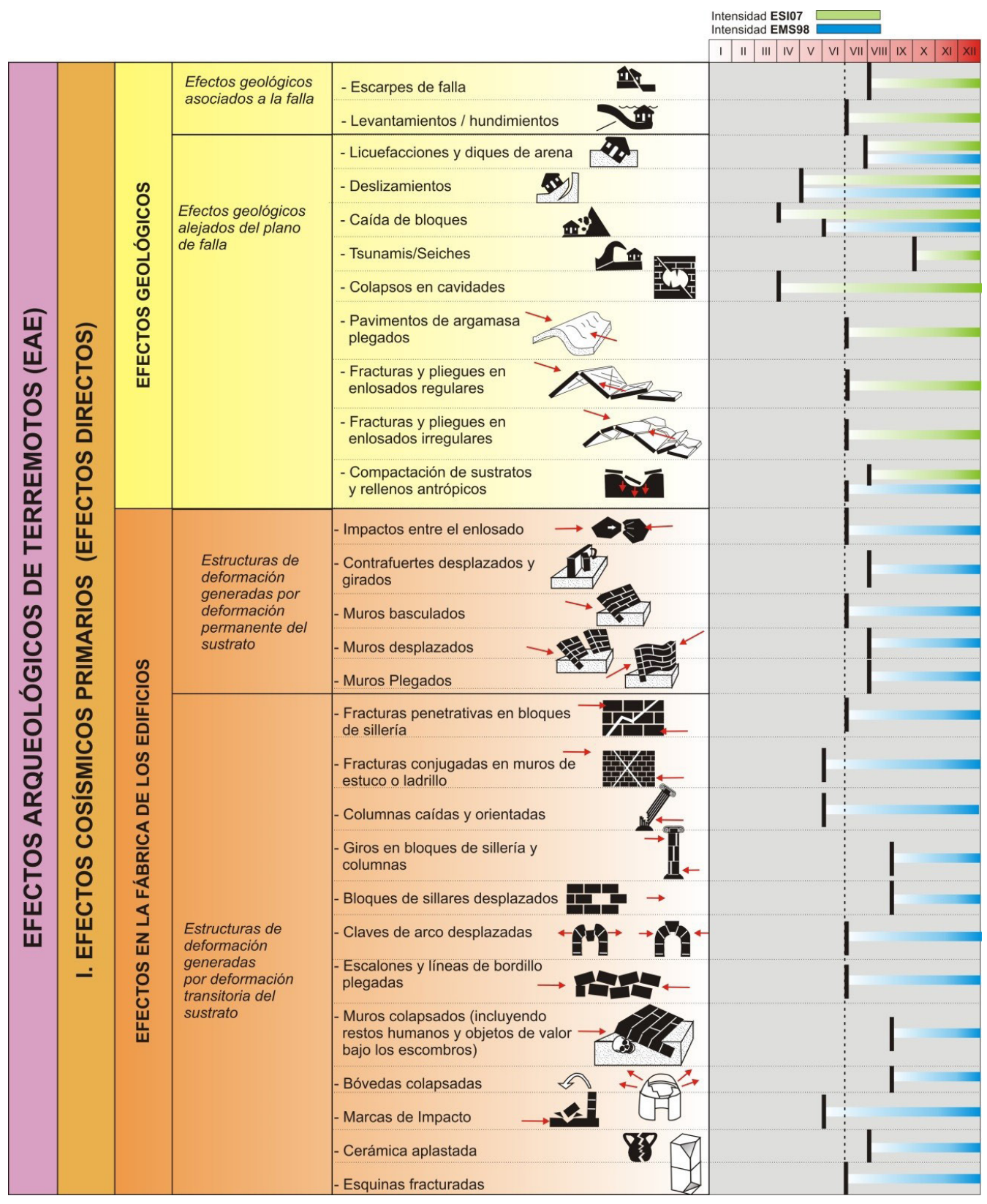

Fig. 3. Tabla de clasificación de Efectos Arqueológicos de Terremotos (Earthquake Archaeological Effects, EAEs) (modificada de Rodríguez-Pascua et al., 2011) e intensidades arqueosismológicas mínimas en las que se producen los EAEs. 


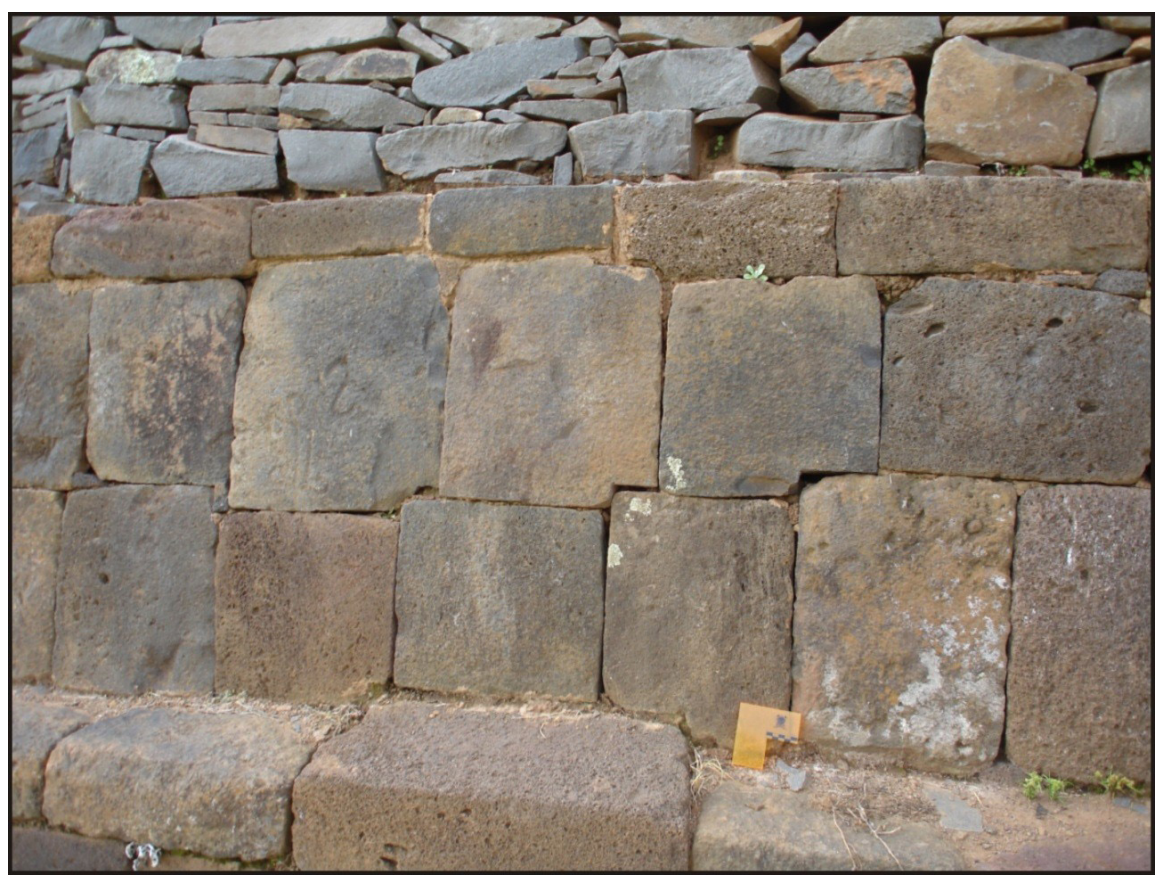

Figura 4. Engatillados antisísmicos en las Yácatas de Tzintzuntzan (Cultura Purhépecha o Tarasca), lago de Pazcuaro (Michoacán, México). Estos impiden el desplazamiento horizontal entre bloques de sillería provocado por terremotos.

Una aplicación importante de los EAEs es la estimación de la intensidad sísmica mínima que los puede generar. Estos efectos se pueden dividir en geológicos y generados en la fábrica de los edificios. Los primeros fueron reducidos en el desarrollo de la escala de intensidades EMS98, pero ampliamente diferenciados y desarrollados por la escala macrosísmica ESI07 (Michetti et al., 2007). Por este motivo, en la escala de intensidades de EAEs se ha utilizado la ESI07 para asignar intervalos de intensidad a los EAEs relacionados con efectos geológicos, como puedan ser licuefacciones, deslizamientos, etc. Todos los efectos geológicos Ilevados a su límite máximo pueden producir la destrucción total de un yacimiento arqueológico o de un edificio patrimonial, por lo que el límite máximo del intervalo de intensidades va a ser siempre XII para todos los EAEs. El límite mínimo se ha fijado utilizando la escala ESI07 y asignando la intensidad más baja a la intensidad menor en la que un efecto geológico pueda quedar preservado en el registro geológico y/o arqueológico. En el caso de deslizamientos, caídas de bloques, compactaciones de rellenos antrópicos y licuefacciones se han podido establecer los límites mínimos tanto para la EMS98 como para la ESI07, siendo diferente para las dos escalas tan solo en el caso de rellenos y caídas de bloques. Para el segundo caso la intensidad mínima es de VI para la EMS98 y de IV para la ESIO7 (Fig. 3). 
En el caso del establecimiento de los valores mínimos de intensidades para los efectos en la fábrica de los edificios se utiliza íntegramente la escala EMS98. Para establecer estos valores mínimos se tienen en cuenta el tipo constructivo, cuyo número se ha reducido para el caso de construcciones históricas y patrimoniales, así como para yacimientos arqueológicos, quedando los siguientes tipos constructivos a tener en cuenta: piedra suelta o canto rodado (al hueso), adobe, mampuesto, ladrillo y sillería (Fig. 3).

Los efectos geológicos se subdividen en efectos asociados al plano de falla que generó el terremoto, o alejados de éste (Fig. 3). El efecto extremo sería la rotura de un yacimiento por el salto de falla generado durante el terremoto. Otros efectos geológicos asociados al plano de falla serían los hundimientos o elevaciones cosísmicas. Para poder identificar estos movimientos en el pasado arqueológico es necesario contar con niveles de referencia, como es el nivel del mar o el de masas lacustres.

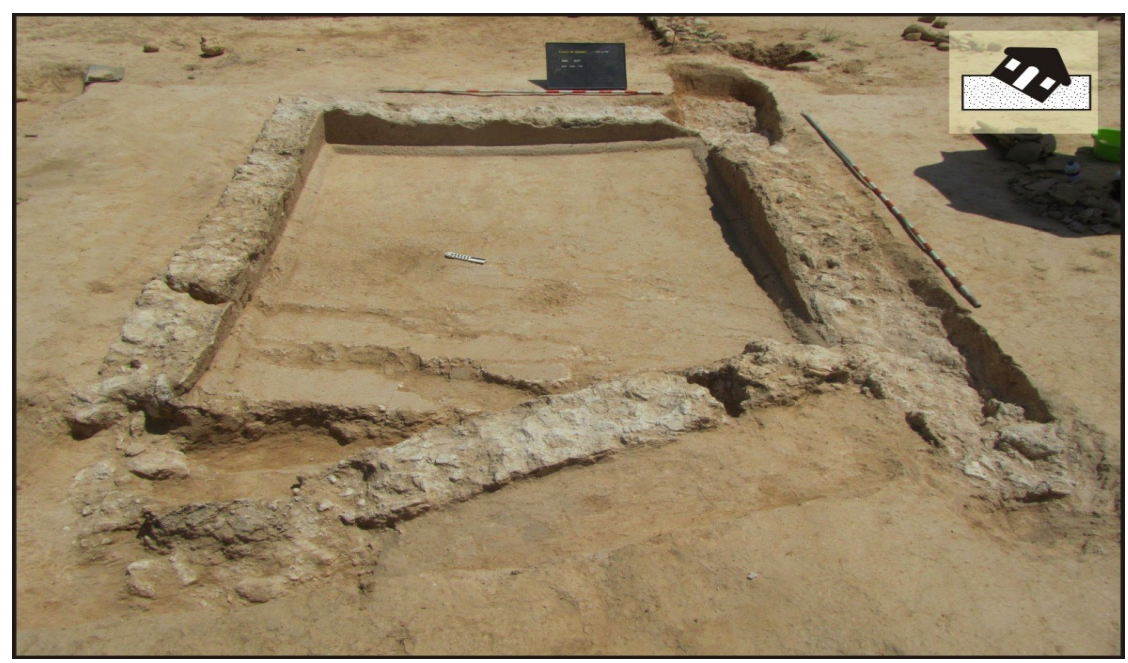

Figura 5. Licuefacción afectando a cisterna hidráulica en el yacimiento romano de La Magdalena (Complutum, Alcalá de Henares, España). Efecto cosísmico geológico primario.

Los efectos geológicos alejados del plano de falla pueden estar asociados a fenómenos como la licuefacción, caída de bloques de roca, tsunamis, colapsos en cuevas o deformaciones en los pavimentos (Fig. 3). Su origen es claramente geológico y el responsable de la deformación en el yacimiento es directamente dicho fenómeno. Uno de los ejemplos más espectaculares, a la vez que dañinos y efímeros en cuanto a su conservación, son las licuefacciones. Éstas consisten en que el sustrato formado por sedimentos no consolidados empapados en agua se comportan durante un breve espacio de tiempo como un fluido. Lo cual puede generar tanto subsidencias, como daños directos en la cimentación de las edificaciones. El fenómeno de la licuefacción ha sido ampliamente documentado 
en la literatura histórica referente a grandes terremotos como en el terremoto de Calabria de 1786 (Lyell, 1872). Un ejemplo son las deformaciones generadas por licuefacción sísmica en el yacimiento romano de La Magdalena (Complutum, Alcalá de Henares) donde estas intrusiones llegan a fracturar los cimientos de una cisterna hidráulica, fracturas por las que se produjeron importantes inyecciones de arena (Fig. 5) (Rodríguez-Pascua et al., 2015a).

Los efectos en la fábrica de los edificios (Fig. 3) se subdividen en los que están asociados a deformaciones permanentes del sustrato y/o cimentaciones de las construcciones y los que están generados por la deformación transitoria del sustrato al paso de las ondas sísmicas. Por tanto, en el caso de los primeros podremos observar tanto las deformaciones del sustrato como las de la edificación; mientras que en los segundos sólo observaremos los daños producidos en la fábrica del edificio.

Un buen ejemplo de las deformaciones permanentes del sustrato son los choques de baldosas que generan rotura de parte de la baldosa, indicando la orientación de la dirección de impacto. Un representativo conjunto de estos efectos se pueden observar en el yacimiento arqueológico de la ciudad romana de Baelo Claudia (Cádiz); así como pliegues en los enlosados, tanto regulares como irregulares (Fig. 6). Esta ciudad fue afectada por dos terremotos en 40-60 AD y 350-395 $\mathrm{AD}$, siendo abandonada en el último terremoto, lo que ha hecho que permanezca prácticamente sin ocupaciones posteriores hasta nuestros días (Silva et al., 2005 y 2009). Esto hace de Baelo Claudia un verdadero laboratorio natural para el estudio de la arqueosismología.

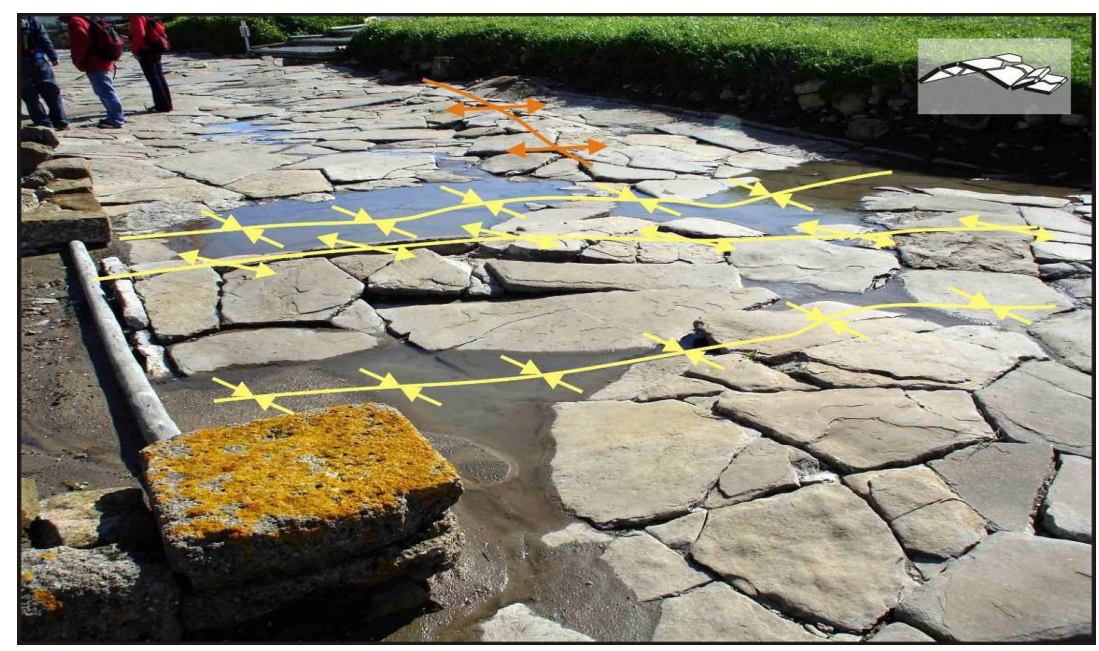

Figura 6. Pliegues (anticlinales y sinclinales) en el enlosado irregular del Decumanus Maximus de Baelo Claudia (Cádiz), deformado por el terremoto que destruyó la ciudad en 350-395 AD. Los pliegues representados en amarillo son perpendiculares a la pendiente topográfica y asociados a dicho terremoto. Los de color naranja son paralelos a la pendiente y están asociados a fenómenos de ladera. Efecto cosísmico geológico primario. 
Los EAEs asociados a la deformación transitoria del sustrato afectan a toda la fábrica del edificio o elemento constructivo, por lo que hay un amplio catálogo de estructuras (Fig. 3). Se describirán aquí las más interesantes desde un punto de vista de su análisis y de la información que pueden aportar. Un EAE muy interesante son las caídas orientadas de columnas, ya que estas nos van a marcar tanto la dirección como el sentido del pulso sísmico predominante. Existen muchos ejemplos en la antigüedad como el Templo de Zeus en Olimpia, la ciudad nabatea de Petra (Fig. 7) o las columnatas de Susita en Israel.

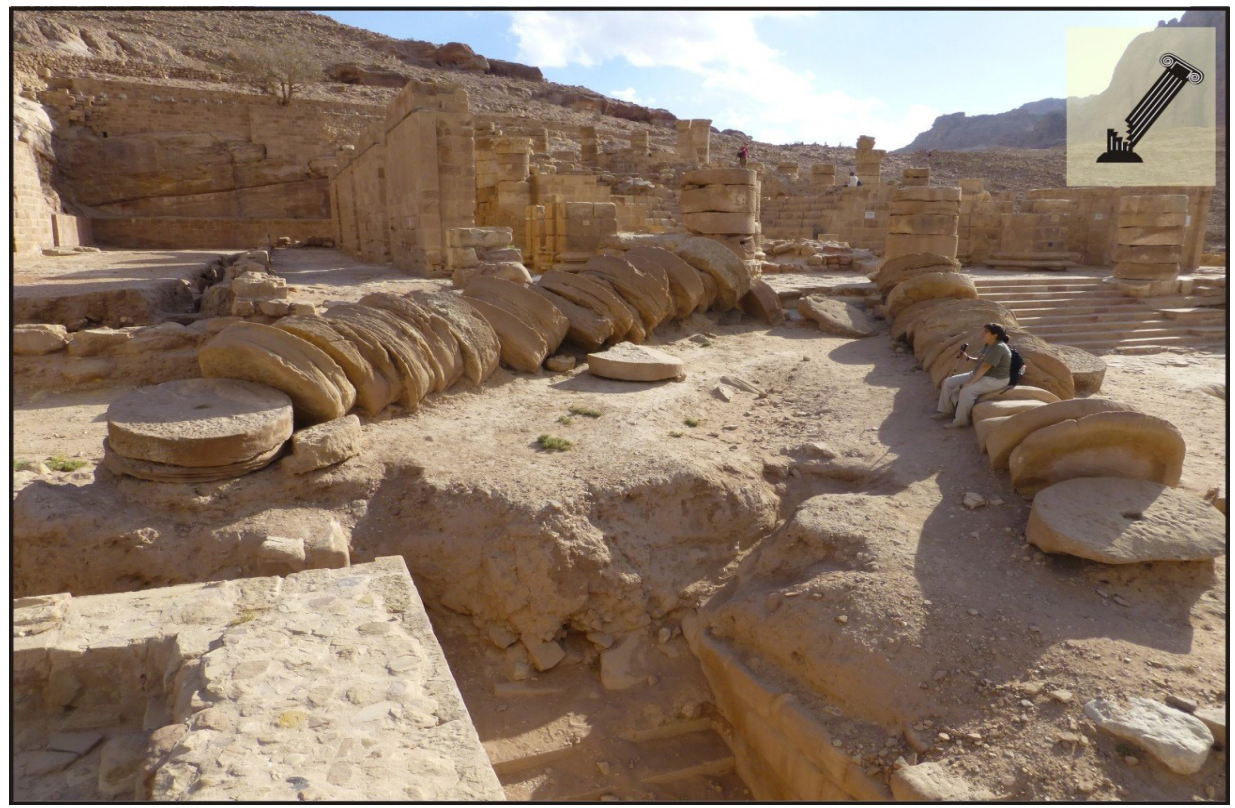

Figura 7. Caída orientada de columnas en el Gran Templo romano de la ciudad nabatea de Petra (Jordania). Efecto cosísmico primario en la fábrica del edificio, son estructuras de deformación generadas por deformación transitoria del sustrato.

Otro elemento EAE clásico es la caída de las claves de los arcos. Éste se produce porque los esfuerzos que sustentan los arcos, que recaen en las paredes laterales, quedan anulados al moverse de forma alternante en la misma dirección que el arco, descargan los esfuerzos de las dovelas del arco; haciendo que las claves caigan por el peso que sustentan dichos arcos. Se pueden generar tanto en bloques de sillería como en arcadas de ladrillo. Buenos ejemplos los podemos encontrar en el Coliseo de Roma, afectado por varios terremotos, que destruyeron la mitad del edificio, debido a que se asentaban sobre materiales no consolidados, mientras que la mitad que está completa se cimienta sobre roca consolidada. Los sedimentos no consolidados amplifican la onda sísmica y aumentan los daños, lo que hizo colapsar la mitad del Coliseo (Fig. 8). 


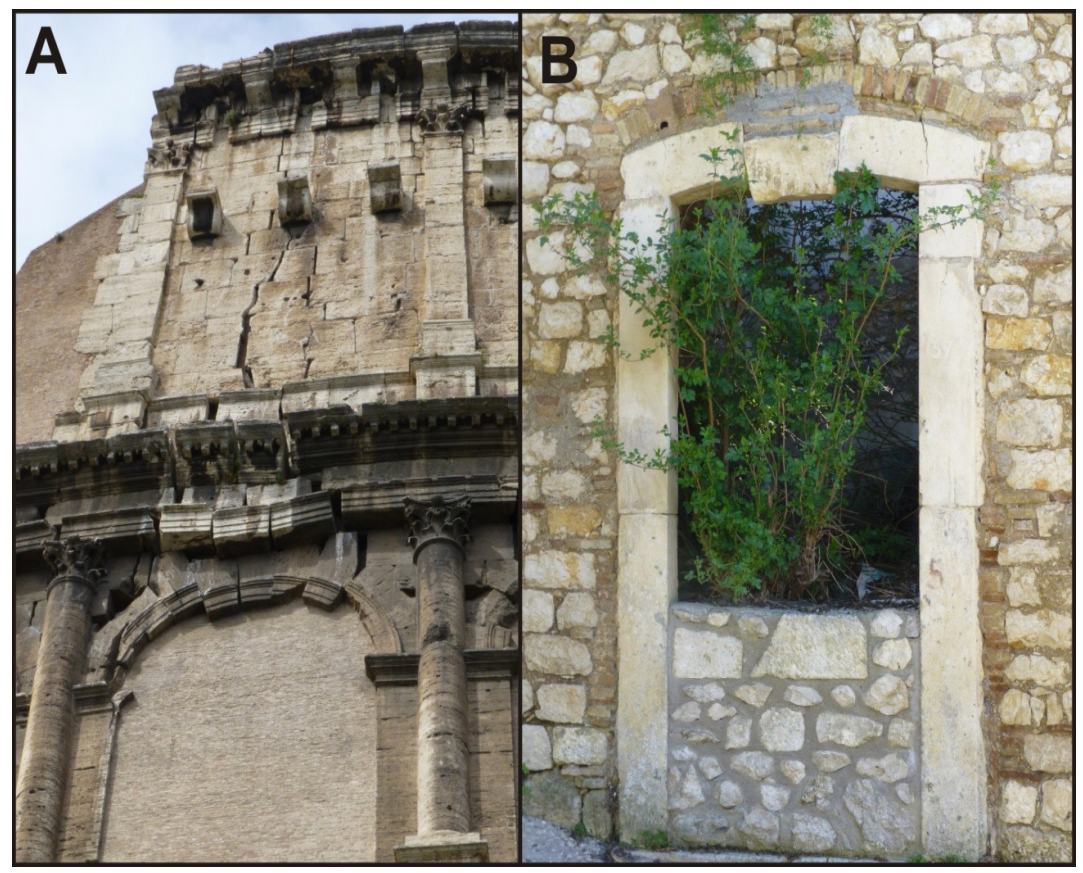

Figura 8. Caída de claves de arco: A) Coliseo de Roma dañado por el terremoto de 1349; B) casa de la localidad de Pecina afectada por el terremoto de Fucino de 1915 (Región de los Abruzos, Italia).

Los pliegues y deformaciones en escalones y bordillos marcan muy bien las direcciones de compresión y en algunos casos incluso reflejan las ondulaciones a las que fueron sometidos durante el paso de las ondas sísmicas. Estas oscilaciones pueden generar la extrusión hacia fuera de los escalones, fenómeno que no se puede dar por otras causas, por lo cual es utilizado como un buen marcador sísmico. Los bordillos de las aceras del decumanus máximo de la ciudad romana de Jerasa en Jordania presentan este tipo de deformaciones a lo largo de todo su trazado, marcando pliegues de ejes paralelos (Fig. 9). Este yacimiento ha sido afectado por terremotos desde tiempos históricos, asociados a la falla del Mar Muerto, siendo el último en 1927, que generó importantes daños en la capital Aman y en las reconstrucciones del yacimiento que se realizaron a principios del s. XX.

Un EAE interesante son las fracturas en esquinas (dipping broken corners), generadas durante la oscilación de bloques de sillería unos con respecto a otros al paso de las ondas sísmicas. Esto hace que el bloque llegue a apoyarse solo sobre las esquinas de forma alternante, produciendo la rotura de estas. En el caso de la columnas es muy útil, ya que al ser de sección circular no están condicionadas por anisotropías previas como los bloques de sillería. De este modo se pueden 
encontrar fracturas en esquinas en los contactos entre fustes y basas o capiteles de columnas, como es el caso del Patio de los Leones en la Alhambra (Fig. 10), donde presentan claras orientaciones E-O (Rodríguez-Pascua et al., 2015b).

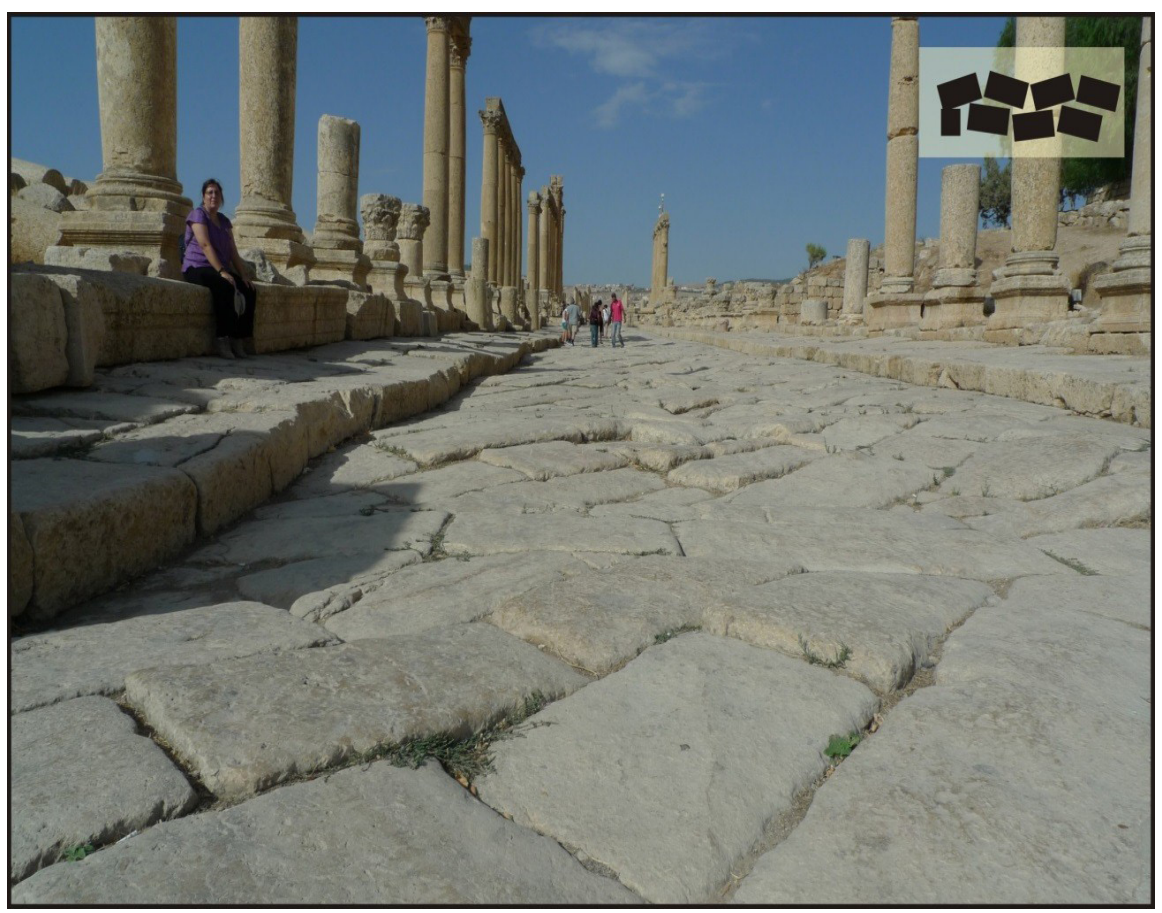

Figura 9. Bordillo y enlosado plegados del decumanus máximo de la ciudad romana de Jerasa (Jordania). Efecto cosísmico primario en la fábrica del edificio, son estructuras de deformación generadas por deformación transitoria del sustrato.

\section{Análisis estructural geológico de EAEs}

Las deformaciones producidas en un conjunto urbano van a estar condicionadas por el mecanismo disparador que las generó. El colapso de un muro puede ser generado tanto por un terremoto como por una explosión, por ejemplo, pero, ¿Cómo podríamos diferenciarlas?. Las deformaciones producidas por un terremoto van a estar condicionadas por la orientación del pulso sísmico predominante, mientras que una explosión generaría el colapso indiscriminado y radial de las estructuras que lo rodeasen. De este modo, si medimos las orientaciones de las deformaciones de la arruinada ciudad de Belchite (Zaragoza), destruida por fuertes combates durante la Guerra Civil Española (septiembre de 1937), no podríamos encontrar ningún patrón de deformación que se ajustase a una orientación en concreto, sino que las deformaciones producidas no estarían orientadas. Sin 
embargo, la llegada del pulso sísmico predominante sí que produce un primer impulso en una determinada orientación, la cual va a condicionar la disposición de las deformaciones. Nosotros proponemos un problema inverso, en el que mediante las deformaciones observadas se puedan calcular las orientaciones del elipsoide de deformación producido por el terremoto en la construcción antigua. La orientación de los ejes de deformación de las diferentes estructuras estudiadas debería ser similar si hubiesen estado generadas por un único efecto, en este caso la llegada del pulso sísmico predominante. En caso contrario, encontraremos una fuerte dispersión en los ejes de los diferentes elipsoides calculados, como sería el caso de estudiar las distintas explosiones generadas por impactos de artillería en una ciudad devastada.

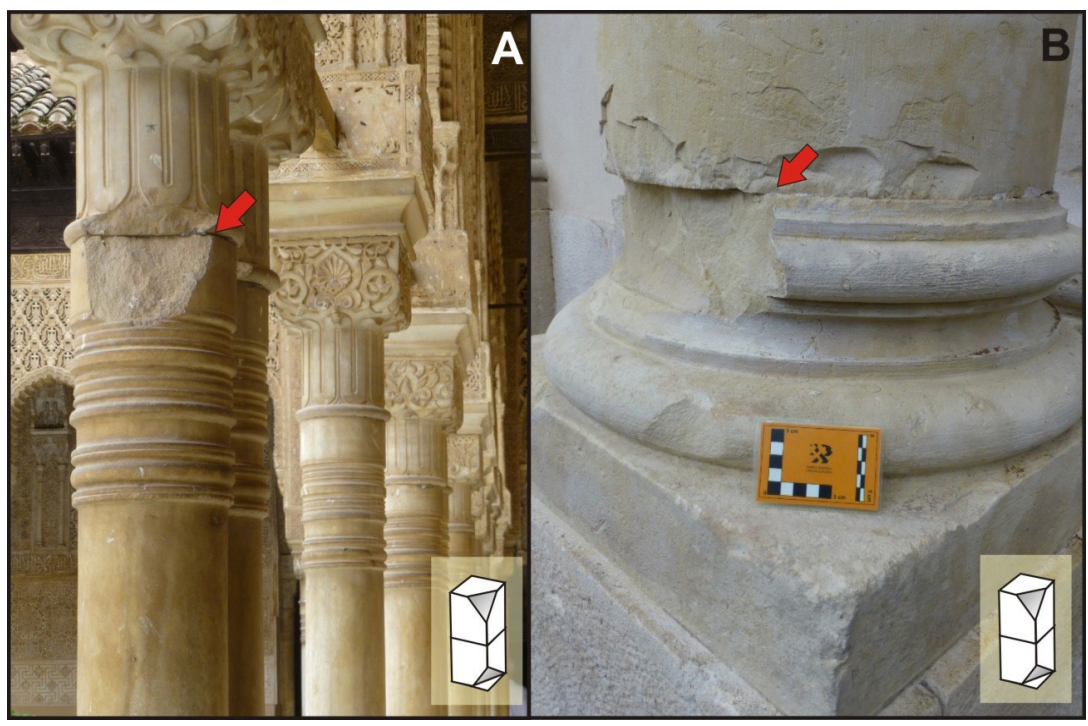

Figura 10. A) Esquinas fracturadas en los fustes y capiteles del Patio de los Leones, Alhambra de Granada (España). B) Análogo actual en la basa de una columna del Palacio Ardinghelli del L’Aquila (Italia) producido por el destructivo terremoto de 2009.

Las técnicas clásicas de análisis estructural geológico son herramientas adecuadas para el cálculo de los elipsoides de deformación de origen sísmico que se extraen de las deformaciones halladas en yacimientos arqueológicos. El yacimiento arqueológico de la antigua ciudad romana de Baelo Claudia (Cádiz) fue el primer yacimiento arqueológico en España estudiado desde un punto de vista arqueosismológico (Silva et al., 2005; 2009). La riqueza y variedad de estructuras de deformación en este yacimiento ha hecho que se haya utilizado como laboratorio para la aplicación de técnicas de análisis estructural geológico (Giner Robles et al., 2009 y 2011). Otro yacimiento estudiado y del que se ha determinado un fuerte terremoto en época alto imperial romana es el Tolmo de Minateda (Albacete) (Rodríguez-Pascua et al., 2009 y 2010). 
Una vez clasificados los EAEs, siguiendo la clasificación propuesta, se pasa al análisis estructural geológico de las deformaciones. Un tensor define la variación en el espacio de la magnitud de los vectores de una determinada propiedad, por lo que con estos análisis conseguiremos calcular los tensores de deformación que sintetizan las características de las deformaciones en construcciones patrimoniales. Un ejemplo de obtención directa de un vector orientado es cuando tenemos un punto que podemos identificar en el estado no deformado y en el deformado, como un bloque de sillería desplazado, en el que podríamos identificar un punto en un bloque inferior y su homólogo en el superior, pudiendo trazar un vector que los una. Este vector va a tener una orientación, que es una dirección en el espacio, con un sentido de movimiento y una magnitud, que corresponde al desplazamiento medido (Fig. 11). Este es uno de los casos más evidentes; en otras situaciones tendremos que recurrir al análisis de la fracturación, en los cuales se pueden desarrollar fracturas conjugadas (en aspa).

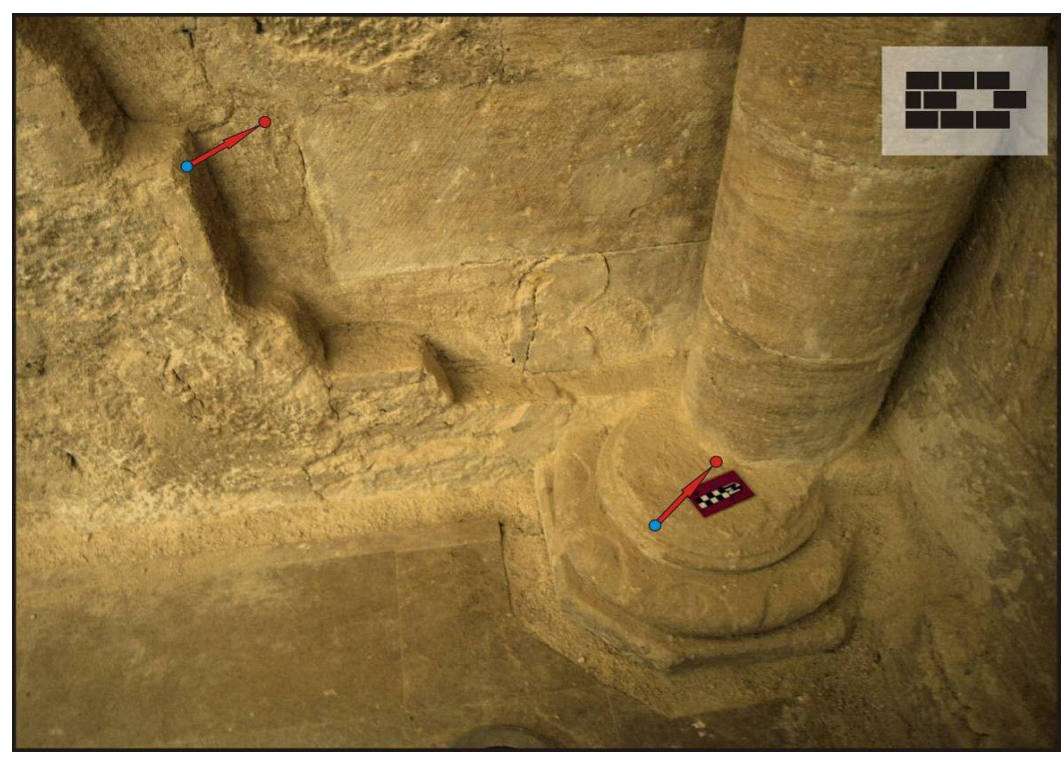

Figura 11. Vector de desplazamiento de la esquina NO de la Torre del Espolón en el Castillo de Lorca, como consecuencia del terremoto de Lorca del 11 de mayo de 2011.

Las fracturas en aspa generadas en muros de ladrillo se ajustan a estos modelos y el análisis de los mismos nos llevará al cálculo de dicho tensor. En el caso de contar con varias orientaciones en el espacio en un mismo edificio, se puede llegar a calcular el elipsoide de deformación, que será compatible con los tensores calculados de forma local. Si proyectamos sobre la horizontal los ejes principales de la deformación podremos calcular las trayectorias de deformación que afectaron a un edificio en concreto o a una ciudad o conjunto de ciudades. Esto hace que podamos comparar estas trayectorias con las principales fallas ac- 
tivas de la zona y ayudarán a corroborar o descartar los posibles efectos sísmicos sobre las edificaciones. El caso del terremoto de Lorca de 2011 es un excelente ejemplo de aplicación de estas metodologías, pudiendo calcularse estas trayectorias para edificaciones tan importantes como la Colegiata de San Patricio, ya afectada por terremotos anteriores (Giner Robles et al., 2012; Rodríguez-Pascua et al., 2012).

\section{Efectos del estudio de los EAEs a la conservación del patrimonio cultural}

Como se ha expuesto en apartados anteriores, los EAEs no se forman de manera aleatoria sino que siguen patrones de deformación condicionados por la llegada del pulso sísmico predominante. Esto hace que, si conocemos la presencia de fallas activas en las proximidades de una localidad, podamos llegar a crear un modelo de deformación teórico que podría generar un hipotético terremoto. Además, la geología, mediante la paleosismología, será capaz de saber cuál será el terremoto máximo esperado de dicha falla, por lo que podremos conocer de antemano cuál será la energía máxima que podría liberar. La arqueosismología también puede diferenciarnos distintos terremotos acaecidos en el pasado, ya que si la misma falla ha generado diferentes terremotos, las deformaciones que generó serán las mismas y un mismo EAE se habrá movido varias veces. Esto es lo que se conoce como reactivación de EAEs (Rodríguez-Pascua, et al., 2012) y nos permite determinar una sucesión sísmica mediante la arqueosismología, y asignar los terremotos deducidos a una o varias fallas, dependiendo de sus características.

Todos estos datos, cruzados con la paleosismología, nos da la posibilidad de crear modelos predictivos de deformación en el patrimonio. De este modo, si suponemos una construcción hipotética (Fig. 12) que fuese sometida a las deformaciones producidas por un terremoto generado por una falla conocida, estas deformaciones estarían condicionadas por la orientación de la construcción. Así pues, si esta construcción sufre un movimiento del terreno perpendicular al eje central de la nave, aparecerán caídas de claves de arco en la fachada principal, pero no en las ventanas de la pared perpendicular. También se desarrollarán fracturas conjugadas en esta fachada, mientras que en los muros perpendiculares aparecerán fracturas horizontales. Las caídas de pináculos serán paralelas a la dirección de movimiento, así como las caídas orientadas de muros.

Por este motivo, el estudio de las fallas activas en el entorno de un conjunto patrimonial, en relación a la orientación del mismo, facilita el desarrollo de modelos predictivos de comportamiento sísmico. La propia información arqueosísmica que contiene el edifico también debe ser utilizada para su protección. Estos datos podrán ser útiles tanto en los planes de conservación como en posibles intervenciones de restauración. 


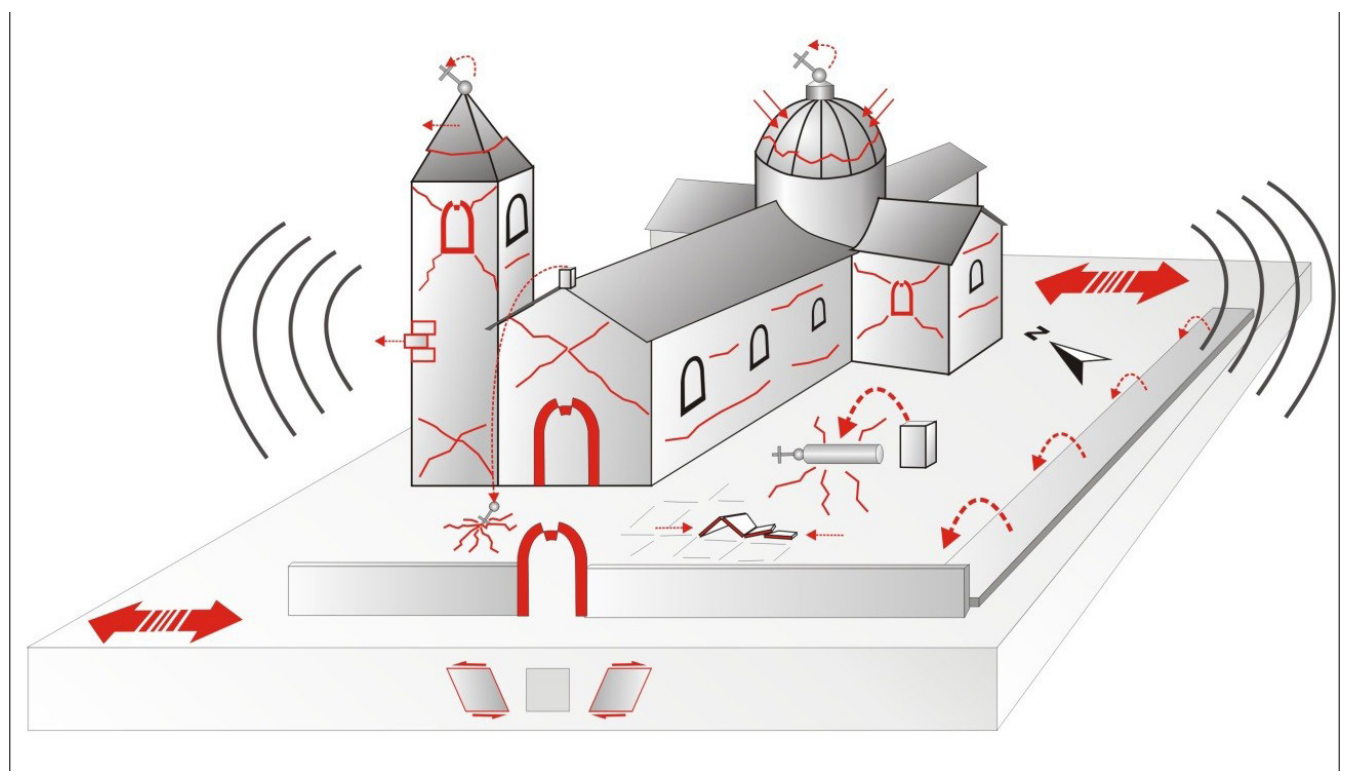

Figura 12. Construcción idealizada con los Efectos Arquitectónicos de Terremotos (EAEs) teóricos que generaría un movimiento sísmico con un desplazamiento del sustrato en dirección NO-SE.

\section{Conclusiones}

La arqueosismología es una técnica multidisciplinar que permite tanto inventariar terremotos no registrados históricamente, como predecir cuál será el comportamiento del patrimonio ante un terremoto. Esto es posible gracias a la clasificación de EAEs y su análisis estructural geológico, con el que obtendremos los elipsoides y trayectorias de deformación de un conjunto patrimonial. Combinando la arqueosismología con la paleosismología se pueden calcular los terremotos máximos esperados para una zona y conocer de qué forma podrán afectar al patrimonio, lo cual podrá ser incluido en los planes de actuación contra el riesgo y proteger nuestro patrimonio.

\section{Agradecimientos}

Este trabajo ha sido financiado por los proyectos IGME CATESI-07 y del MINECO CGL2012-37281-C02.01 (USAL). Es una contribución del Grupo de trabajo QTECT-AEQUA. 


\section{Bibliografía}

J.D. DE LA HOZ. "Terremoto en Lorca: consecuencias y actuaciones sobre el patrimonio religioso". Patrimonio Cultural de España, 6 (2012a): 107-122.

J.D. DE LA HOZ. "Efectos del terremoto de Lorca del 11 de mayo de 2011 sobre el patrimonio religioso. Análisis de emergencia y enseñanzas futuras". Boletín Geológico y Minero, 123 (4) (2012b): 515-536.

J. GALBIS RODRÍGUEZ. Catálogo Sísmico de la zona comprendida entre los meridianos $5^{\circ} \mathrm{E}$ y $20^{\circ} \mathrm{W}$ de Greenwich y los paralelos $45^{\circ}$ y $25^{\circ}$ Norte, Tomo I. Instituto Geográfico Catastral y de Estadística, Madrid (1932), 818 pp.

J. GALBIS RODRÍGUEZ. Catálogo Sísmico de la zona comprendida entre los meridianos $5^{\circ} \mathrm{E}$ y $20^{\circ} \mathrm{W}$ de Greenwich y los paralelos $45^{\circ}$ y $25^{\circ}$ Norte, Tomo II. Instituto Geográfico Catastral y de Estadística, Madrid (1940), 280pp.

J.L. GINER-ROBLES, M.A. RODRÍGUEZ-PASCUA, R. PÉREZ-LÓPEZ, P.G. SILVA, T. BARDAJÍ, C. GRÜTZNER, Y K. REICHERTER. Structural Analysis of Earthquake Archaeological Effects (EAE): Baelo Claudia Examples (Cádiz, South Spain). Instituto Geológico y Minero de España (2009), 130 pp.

J.L. GINER-ROBLES, P.G. SILVA BARROSO, R. PÉREZ-LÓPEZ, M.A. RODRÍGUEZ-PASCUA, T. BARDAJÍ AZCÁRATE, V.H. GARDUÑO-MONROY, Y J. LARIO GÓMEZ. Evaluación del daño sísmico en edificios históricos y yacimientos arqueológicos. Aplicación al estudio del riesgo sísmico. Proyecto EDASI. Serie Investigación. Fundación MAPFRE (2011), 96 pp.

J.L. GINER-ROBLES, R. PÉREZ-LÓPEZ, P.G. SILVA BARROSO, M.A. RODRÍGUEZ-PASCUA, F. MARTÍN-GONZÁLEZY L. CABAÑAS. Análisis estructural de daños orientados en el terremoto de Lorca del 11 de mayo de 2011. Aplicaciones en Arqueosismología. Boletín Geológico y Minero, 123 (4) (2012): 503-513.

E.GUIDOBONI. I terremoti prima del Mille in Italia e nell'area Mediterranea: storia, archaeologia, sismologia. Bologna. SGA-Instituto Nazionale di Geofisica (1989). 765 pp.

IPCE. Patrimonio en riesgo: seismos y bienes culturales. Patrimonio Cultural de España, 6 (2012), 268 pp.

R. LANCIANI,. Segni di Terremoti negli edifizi di Roma Antica. Bull. Della Comm. Arch. Communale Roma (1918), 1-30.

LYELL, C. The Principles of Geology. The modern changes of the earth and its inhabitants. London (1872). Vol. II, 456pp.

J.M. MARTíNEZ-SOLARES Y J. MEZCUA. Catálogo sísmico de la Península Ibérica (880 a.C.-1900). Instituto Geográfico Nacional. Ministerio de Fomento (2002), 756 pp. 
F.MARTÍNEZ-VÁZQUEZ. El terremoto de Lisboa y la Catedral de Coria (Vicisitudes del Cabildo) 1755-1759. Colección Temas Cauriaciences. Vol. V. Ed. Ayuntamiento de Coria (1999), 187 pp.

M. MUÑOZ CLARES, M. FERNÁNDEZ CARRASCOSA, M.O. ALCOLEA LÓPEZ, M.C. ARCAS NAVARRO, N. ARCAS RUIZ, P. CARO DEL VAS, M.T. CRUZ LÓPEZ, M. GARCÍA POVEDA, M. A. GARCÍA VALERA, B. LLAMAS MARTÍNEZ, Y A. E. RUIZ LLANES. Sismicidad histórica y documentación municipal: el caso de Lorca. Boletín Geológico y Minero, 123 (4) (2012): 415-429.

A. NIKONOV. On the metholology of archaeoseismic research into historical monuments. En: Engineering Geology of Ancient Works, Monuments and Historical Sites (G. Marinos and G. Koukis, Eds.). Balkema, Roterdam (1988), 1325-1320.

G. RAPP. Earthquakes in the Troad. En: Troy: The archaeological Geology (G. Rapp and J.A. Gifford, Eds.). Princenton (1982), 43-58.

M.A. RODRÍGUEZ-PASCUA, L. ABAD CASAL, R. PÉREZ-LÓPEZ, B. GAMO PARRA, P.G. SILVA, V.H. GARDUÑO-MONROY, J.L. GINER-ROBLES, I. ISRADE-ALCÁNTARA, J. BISCHOFF Y J.P. CALVO. Roman, visigoth and islamic evidence of earthquakes recorded in the archaeological site of El Tolmo de Minateda (Prebetic Zone, Southeast of Spain). 1st INQUAIGCP 567 International Workshop on Earthquake Archaeology and Palaeoseismology, 7th- 13th September (2009), 110-114.

M.A. RODRÍGUEZ-PASCUA, P.G. SILVA, V.H. GARDUÑO-MONROY, R. PÉREZ-LÓPEZ, I. ISRADE-ALCÁNTARA, J.L. GINER-ROBLES, J. BISCHOFF $Y$ J.P. CALVO. Ancient earthquakes from archaeoseismic evidence during the Visigothic and Islamic periods in the archaeological site of "Tolmo de Minateda" (SE of Spain). En: Ancient Earthquakes. (M. Sintubin, I.S. Stewart, T.M. Niemi and E. Altunel Eds.). Geological Society of America, Special Paper (2010), 471: 171-184.

M.A. RODRÍGUEZ-PASCUA, R. PÉREZ-LÓPEZ, P.G. SILVA, J.L. GINER-ROBLES, V.H. GARDUÑO-MONROY Y K. REICHERTER. A Comprehensive Classification of Earthquake Archaeological Effects (EAE) for Archaeoseismology. Quaternary International (2011), 242: 20-30.

M.A. RODRÍGUEZ-PASCUA, R. PÉREZ-LÓPEZ, F. MARTÍN-GONZÁLEZ, J.L. GINER-ROBLES Y P.G. SILVA. Efectos arquitectónicos del terremoto de Lorca del 11 de mayo de 2011. Neoformación y reactivación de efectos en el Patrimonio Cultural. Boletín Geológico y Minero, 123 (4) (2012): 487-502

M.A. RODRÍGUEZ-PASCUA, P.G. SILVA, R. PÉREZ-LÓPEZ, J.L. GINERROBLES, F. MARTÍN-GONZÁLEZ, Y B. DEL MORAL. Polygenetic sand volcanoes: On the features of liquefaction processes generated by a single event (2012 Emilia Romagna 5.9 Mw earthquake, Italy). Quaternary International, 357 (2015): 329-335. 
M.A. RODRÍGUEZ-PASCUA, P.G. SILVA, R. PÉREZ-LÓPEZ, J.L. GINER-ROBLES, F. MARTÍN-GONZÁLEZ, M.A. PERUCHA. Preliminary intensity correlation between macroseismic scales (ESI07 and EMS98) and Earthquake archaeological effects (EAEs). 4th International INQUA Meeting on Paleoseismology, Active Tectonics and Archeoseismology (PATA) (2013), 221-224.

M.A. RODRÍGUEZ-PASCUA, C. HERAS, A.B. BASTIDA, J.L. GINER-ROBLES, P.G. SILVA, M.A. PERUCHA, E. ROQUERO, P. CARRASCO, R. PÉREZ-LÓPEZ, J. LARIO Y T. BARDAJI. New insights on the occurrence of ancient earthquakes in Central Spain: Archaeoseismology of the Complutum area (4th century AD, Madrid). Miscellanea, 27 (2015a): 202-405.

M.A. RODRÍGUEZ-PASCUA, M.A. PERUCHA, P.G. SILVA, J.L. GINER ROBLES, R. PÉREZ-LÓPEZ, GARCÍA GUTIÉRREZ, G.B. Evidencias de efectos arqueológicos de terremotos (EAEs) en la Alhambra (Granada, Andalucía, España). XIV Reunión Nacional de Cuaternario, Granada (2015b). Extended Abstracts.

M. SINTUBIN, Y I. S. STEWART. A Logical Methodology for Archaeoseismology: A Proof of Concept at the Archaeological Site of Sagalassos, Southwest Turkey. Bulletin of the Seismological Society of America, 98 (5) (2008): 2209-2230.

P.G. SILVA, F. BORJA, C. ZAZO, J.L. GOY, T. BARDAJÍ, L. DE LUQUE, J. LARIO $Y$ C.J. DABRIO. Archaeoseismic record at the ancient Roman City of Baelo Claudia (Cádiz, south Spain). Tectonophysics, 408 (1-4) (2005): 129-146.

P.G. SILVA, K. REICHERTER, CH. GRÜTZNER, T. BARDAJÍ, J. LARIO, J.L. GOY, C. ZAZO Y P. BECKER-HEIDMANN. Surface and subsurface palaeoseismic records at the ancient Roman city of Baelo Claudia and the Bolonia Bay area, Cádiz (south Spain). Geological Society of London, Special Publication (2009), 316: 93-121.

S. STIROS. Earthquake effects on Ancient Constructions. In: New Aspect of Archaeological Science in Greece (R.E. Jones and H.W. Catling, Eds.). British Schools at Athens, Fitch Occasional Paper (1988a), 3: 1-6.

S. STIROS. Archaeology, a tool to study active tectonics - The Aegean as a case study. Eos, Trans. Am. Geophys. Union. 13 (1988 b): 1636-1639.

S. STIROS Y R.E. JONES. Archaeoseismology. Institute of Geology and Mineral Exploration. Fitch Laboratory Occasional Paper. Stiros S., and Jones, R.E., Eds. Atenas (1996). $268 \mathrm{p}$.

B. ZANG, Y. LIAO, S. GUO, R. WALLACE, R. BUCKHAM, Y T. HANKS. Fault scarps related to the 1739 earthquake and seismicity of the Yinchuan graben, Ningxia Zizhiqu, China. Bull. Soc. America. 76 (1986): 1253-1287. 
